\title{
Academic Publication Management with PUMA - Collect, Organize and Share Publications
}

\author{
Dominik Benz ${ }^{1}$, Andreas Hotho ${ }^{3}$, Robert Jäschke ${ }^{1}$, Gerd Stumme ${ }^{1}$, \\ Axel Halle ${ }^{2}$, Angela Gerlach Sanches Lima ${ }^{2}$, Helge Steenweg ${ }^{2}$, and Sven Stefani ${ }^{2}$ \\ ${ }^{1}$ Knowledge \& Data Engineering Group \\ University of Kassel \& L3S Research Center \\ D-34121 Kassel, Germany \\ \{lastname\} @cs. uni-kassel.de \\ ${ }^{2}$ University Library of Kassel \\ D-34127 Kassel, Germany \\ \{lastname\}@bibliothek.uni-kassel.de \\ ${ }^{3}$ University of Würzburg \\ Data Mining and Information Retrieval Group \\ Am Hubland, D-97074 Würzburg \\ \{lastname\}@informatik.uni-wuerzburg.de
}

\begin{abstract}
The PUMA project fosters the Open Access movement und aims at a better support of the researcher's publication work. PUMA stands for an integrated solution, where the upload of a publication results automatically in an update of both the personal and institutional homepage, the creation of an entry in a social bookmarking systems like BibSonomy, an entry in the academic reporting system of the university, and its publication in the institutional repository. In this poster, we present the main features of our solution.
\end{abstract}

Keywords: Publication Management, Puma, BibSonomy, Open Access, Institutional Repository, Tagging, Bookmarking, Metadata Sharing.

\section{Introduction}

The project „PUMA - Academic Publication Management" ${ }^{\text {“1 }}$ is funded by the German Research Foundation (DFG) and has been started on August 1st, 2009. PUMA is a joint project of the University Library ${ }^{2}$ and the Knowledge \& Data Engineering Group $^{3}$ of the University of Kassel (cf. [2]).

Open Access ${ }^{4}$ is a publication model that allows authors to publish their articles free of charge, and users to freely access them. The costs are borne by the institution that is providing the institutional repository. There are several reasons for this

\footnotetext{
${ }^{1}$ http://puma.uni-kassel.de/

${ }^{2}$ http://www.ub.uni-kassel.de/

${ }^{3}$ http://www.kde.cs.uni-kassel.de/

${ }^{4}$ http://www.open-access.net/
} 
publication model. With reduced budgets and increased costs for journals, many university libraries cannot afford the subscription of all relevant journals any longer. Furthermore, Open Access supports a timely publication and broader visibility of articles so that research results can be taken up earlier and by more researchers, decreasing thus the turn around time of scientific results.

Even though many researchers support the open access movement in principle, they often do not contribute their publications to the institutional repository of their university. Key reasons are that they do not see an immediate benefit from this additional effort, and that the upload is not integrated in their usual workflow. PUMA aims therefore for an integrated solution, where the upload of a publication results automatically in an update of both the personal and institutional homepage, the creation of an entry in the social publication sharing platform BibSonomy, ${ }^{5}$ an entry in the academic reporting system of the university, and its publication in the institutional repository. At the time of upload, metadata from several data sources will be collected automatically in order to support the user. In addition, PUMA aims to provide a publication management platform for all researchers and students to be used on a daily basis, which reduces not only the open access publication effort but also the effort to manage one's own publications.

The PUMA is hosted by the University Library. It implements state-of-the-art Web 2.0 functionality. The platform includes all features known from BibSonomy, like tagging of publications, easy usage, an API and scalability. As a showcase, PUMA will be integrated with the open access repository platform DSpace, the library system PICA, the Typo3 content management system, and BibSonomy. The system is open for adaptation to other standards and systems. The project results will be published as open source software. This implies that any university resp. university library will be able to build its own publication management according to individual needs.

\section{Architecture}

PUMA is based on the well-known social bookmarking system BibSonomy, which allows users to organise and share bookmarks and publications in a collaborative manner. The basic building blocks of PUMA are an Apache Tomcat servlet container using Java servlet technology and a MySQL database as backend. All search engine like requests are handled by an adopted Lucene framework. A detailed description can be found in [1].

\section{User Interface}

The user interface is depicted in Fig.1, which shows bookmarks, publication posts and tags of a user. The page is divided into four parts: the header (showing information such as the current page title and location, navigation links and search boxes), two

\footnotetext{
${ }^{5}$ http://www.bibsonomy.org/
} 
lists of posts - one for bookmarks and one for publications - each sorted by date in descending order, and a list of tags related to the posts.

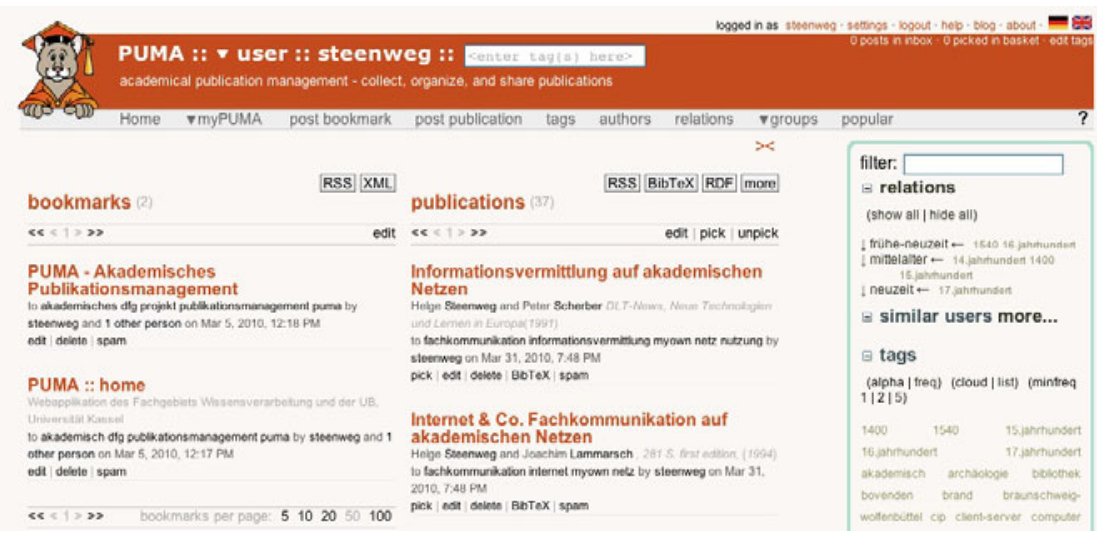

Fig. 1. PUMA displays bookmarks, publication metadata and tags simultaneously

\section{Features}

All possibilities known from BibSonomy are included within PUMA. In addition, there are some more advanced features: Apart from standard folksonomy features such as an intuitive user interface, navigation along all dimensions, or browser integration via RSS feeds, PUMA provides tag hierarchies, group management and privacy features, and numerous import and export functions, in particular to and from BibTeX, EndNote, and Zotero.

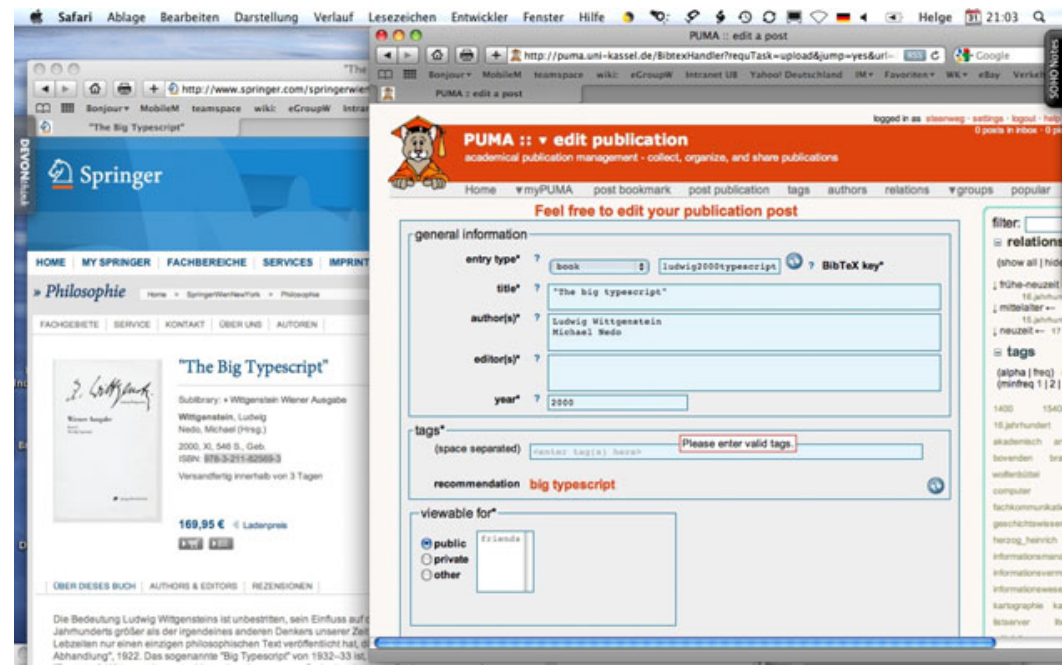

Fig. 2. Using the PUMA-Browser-Plugin to store the publication metadata for an ISBN 


\section{Posting a Publication}

It is very simple to add publications by entering their metadata manually into the form fields of PUMA. But there are several less laborious ways to get data into PUMA, e.g., file import from BibTeX oder EndNote - or even more conveniently by using a special browser plugin. Every user can then store publication entries while browsing the web. This is accomplished by so-called scrapers for over 70 portals (e.g., Amazon, IEEE, Scopus, Muse, BioMed, JSTORE, arXiv, etc.) or library catalogs. Another option is to highlight an ISBN, ISSN or DOI phrase on a web page and to click the postPublication-button of the plugin. This triggers the upload of the associated data to PUMA (s. Fig. 2). All data in PUMA can be edited and exported in over 30 formats. In addition, custom export formats can be created and uploaded by each user.

\section{CV}

Another common requirement of authors is to have an easy-to-maintain CV page, featuring all personal details and publications. Within PUMA every user can generate his own CV using a variety of import and export formats. Especially for users of Typo3, a plugin exists with adjustable parameters to maintain an automatically created $\mathrm{CV}$ an its own homepage.

\section{Next Steps}

Currently, we are working on a path to integrate the data automatically into the workflow of an institutional repository, e.g. DSpace. To this end, we implement the SWORD protocol ${ }^{6}$ for easier content exchange between repositories.

Future work also includes better support for research groups (e.g., hierarchical group structures, extended administration options, custom tag sets), reporting functionalities, and a framework for system tags. The improvement of the user interface and the help system are an ongoing effort.

A beta version of PUMA is already available at http://puma.uni-kassel.de/. The rollout to all members of the University of Kassel is planned for summer 2010. The system will be made available under an open source licence at the end of the project.

\section{References}

1. Benz, D., Hotho, A., Jäschke, R., Krause, B., Mitzlaff, F., Schmitz, C., Stumme, G.: The social bookmark and publication management system BibSonomy. VLDB Journal (to appear)

2. Steenweg, H.: Publikationsmanagement mit PUMA auf der Basis von BibSonomy, http: //www. opus-bayern.de/bib-info/volltexte/2010/865/

\footnotetext{
${ }^{6} \mathrm{http} / / / \mathrm{www}$. swordapp.org/
} 